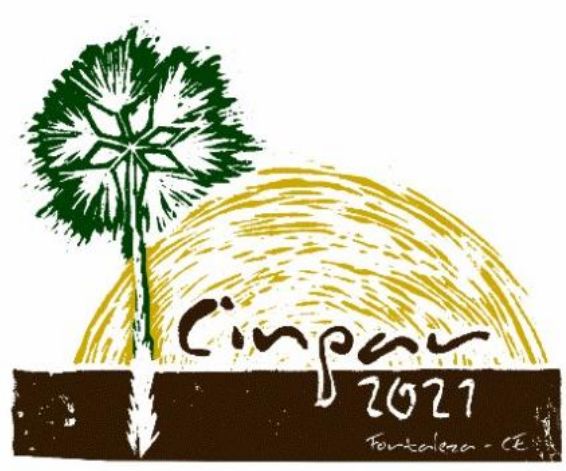

XVII Congresso Internacional sobre Patologia e Reabilitação das Construções

XVII Congreso Internacional sobre Patología y Rehabilitación de las Construcciones

XVII International Conference on Pathology and Constructions Rehabilitation

FORTALEZA (Brasil), 3 a 5 de junho de 2021

https://doi.org/10.4322/CINPAR.2021.010

\title{
Fluidez de pastas autocompactantes con polvo de basalto
}

\section{Flowability of self-compacting pastes with basalt powder}

\author{
Daniel Pizzutti ${ }^{1}$, Jorge Velázquez ${ }^{1}$, Yanina Flores ${ }^{1}$ \\ ${ }^{1}$ Universidad Nacional de Misiones, Laboratorio de Ingeniería Civil, Oberá, Argentina, pizzutti@fio.unam.edu.ar
}

\begin{abstract}
Resumen: Un hormigón autocompactante requiere elevados contenidos de material fino para mantener la estabilidad de la mezcla, garantizando una elevada fluidez y una moderada viscosidad. El objetivo del trabajo es analizar la viabilidad de utilización del polvo de basalto (PB) como material suplementario al cemento Portland para proveer la cantidad adecuada de partículas finas en pastas autocompactantes como la primera fase del diseño de morteros y hormigones. Se caracterizaron muestras de PB provenientes de cuatro canteras de la Provincia de Misiones con el fin de utilizarlas en la elaboración de pastas con un cemento Portland compuesto. El estudio de las pastas abarcó la determinación del escurrimiento con cono de Kantro y la velocidad de flujo en cono de Marsh. Los resultados indican que es viable la utilización del PB desde el enfoque de autocompactabilidad, teniendo en cuenta que la relación entre agua y material fino es determinante en el diseño de la mezcla, afectando principalmente la demanda y el punto de saturación del aditivo y la fluidez y viscosidad de las pastas autocompactantes con diferentes porcentajes de reemplazo con PB. La resistencia a compresión de las pastas evaluada a 28 días varió entre 55 y $25 \mathrm{MPa}$ para reemplazos crecientes de PB de 20 a 50\%, respectivamente.
\end{abstract}

Palabras claves: fluidez, pasta autocompactante, polvo de basalto, viscosidad.

Keywords: flowability, self-compacting paste, basalt powder, viscosity.

\section{Introducción}

Los avances tecnológicos en cuanto a materiales y procesos de producción han permitido el desarrollo de un hormigón que puede deformarse bajo la acción de su propio peso llenando todos los sectores del encofrado sin necesidad de vibración interna o externa, con capacidad para sortear obstáculos (Fava C. y Fornasier G. (2004)), denominado Hormigón Autocompactante (HAC). Este tipo de hormigón posee varias ventajas sobre el hormigón convencional ( $\mathrm{HC}$ ), incluyendo: nula necesidad de vibración; disminución de tiempos de ejecución y costo de mano de obra; reducción de contaminación sonora; mejoramiento de la capacidad de llenado de encofrados; mejoramiento de la zona de transición entre la pasta de cemento y el agregado o la armadura; disminución de la permeabilidad; mejoramiento de la durabilidad; y aseguramiento de un desempeño estructural adecuado (Shi C. et al. (2015)).

Los HAC se logran con aditivos químicos reductores de agua de alto rango, denominados superfluidificantes (SP), y con altos contenidos de material fino (MF), incluyendo partículas $<0,15 \mathrm{~mm}$ (cemento, adiciones minerales y polvo). Considerando que el HAC en estado fresco puede ser analizado como una suspensión de partículas se pueden definir distintas fases de análisis: el hormigón como mezcla de agregado grueso y mortero, el mortero como mezcla de agregado fino y pasta y la pasta como mezcla de MF, agua y aditivo SP. 
Un HAC en estado fresco presenta una alta fluidez y una moderada viscosidad para asegurar la estabilidad del conjunto. Para alcanzar estas propiedades es indispensable la utilización de aditivos SP y un contenido de MF mayor al de un hormigón convencional (Tobes J. M. (2009)). Este elevado contenido de MF (entre 450 y $600 \mathrm{~kg} / \mathrm{m}^{3}$ ), lo provee el cemento Portland y la incorporación de adiciones minerales, como escoria de alto horno, filler calcáreo, humo de sílice, entre otras (Fava C. y Fornasier G. (2004)).

La producción de agregado triturado en canteras a cielo abierto en la provincia de Misiones genera importantes volúmenes de polvo de basalto (PB) presente en la arena de trituración (fracción de 0 a 6 mm o binder), la cual es poco utilizada para la elaboración de hormigón estructural (Pizzutti, D. et al. (2016)). Los contenidos de PB como MF ( $<0,15 \mathrm{~mm}$ ) en dicha fracción pueden oscilar entre 18 y $34 \%$ dentro de una misma pila de acopio (Pizzutti, D. et al. (2016)), lo que podría ser una fuente interesante de MF para elaborar HAC; esto coincide con otros autores (Felekoğlu, B. (2008) y Uysal M. y Yilmaz K. (2011)) que sugieren que el uso de arenas ricas en MF puede dar lugar a beneficios técnicos y económicos a los productores de hormigón. Las partículas menores a $0,125 \mathrm{~mm}$ adquieren gran influencia en la trabajabilidad de las mezclas (Cepuritis $R$. et al. (2011)).

Mezclas con polvo de basalto en bajos porcentajes de reemplazo del contenido de cemento han demostrado mejorar la resistencia de pastas y morteros (Dobiszewska M. y Beycioğlu A. (2017)), en contrapartida con altos porcentajes de reemplazo podrían generar una disminución de resistencia por efecto de dilución del cemento (Dobiszewska M. et al. (2019)).

Específicamente estudios sobre pastas de cemento con adición de subproductos de trituración de rocas para desarrollar HAC, muestran evidencias de un comportamiento satisfactorio bajo determinadas condiciones en función de las propiedades del polvo utilizado. La forma y distribución de tamaño de partículas del polvo de trituración requiere una mayor dosis de aditivo SP para lograr similares propiedades de fluidez que mezclas elaboradas con filler calcáreo (Schankosk R. A. et al. (2016)). La incorporación de arena de trituración caliza puede mejorar las características durables de HAC reduciendo levemente la fluidez y manteniendo la estabilidad y la resistencia a la segregación en estado fresco (Benyamina S. et al. (2019)).

Debido a que reología de la pasta depende fuertemente de la superficie específica del MF, se pueden controlar o modificar las propiedades en estado fresco del HAC mediante la cantidad y granulometría del polvo utilizado (Cepuritis R. et al. (2016),).

La incorporación de residuos de polvo de trituración es viable para la elaboración de HAC con propiedades aceptables en estado fresco y endurecido (Mahalingam B. et al. (2018)). Esto da lugar a un hormigón más sustentable con un residuo de bajo costo (Dobiszewska M. (2017)). Algo similar a lo que ocurre con la posibilidad de incorporación de agregados reciclados en HAC (Revilla-Cuesta V. et al. (2020)).

\section{Materiales y metodología}

\subsection{Cacterización de materiales}

Para la elaboración de pastas se utilizó cemento Portland compuesto (CPC40), agua de red, aditivo superfluidificante en base policarboxilato y polvo de basalto (PB) con partículas menores a $150 \mu \mathrm{m}$ obtenido por tamizado en vía seca de variadas muestras de arena de trituración extraídas de canteras de la provincia de Misiones. En la Tabla 1 se indican las muestras de PB utilizadas en el trabajo y su procedencia, incluyendo dos submuestras, una obtenida de un depósito de sedimento de PB producto del lavado por agua pluvial (CEL-Sed) y otra obtenida a partir de un ciclón extractor de polvos (CSM-Cic).

En relación a la caracterización de los materiales, en el caso del cemento se determinó la densidad relativa según IRAM 1624 (2005), la granulometría de partículas y la superficie específica mediante difractometría láser. La densidad relativa del aditivo SP se determinó según IRAM 1663 (2002). 
Tabla 1 - Muestras de PB empleadas

\begin{tabular}{|c|c|c|}
\hline Código & Localidad & Procedencia \\
\hline CEN & San José & Acopio \\
\hline CIE & Profundidad & Acopio \\
\hline CEL & Santa Ana & Acopio \\
\hline CEL-Sed & Santa Ana & Depósito de sedimento \\
\hline CSM & San Martín & Acopio \\
\hline CSM-Cic & San Martín & Ciclón extractor \\
\hline
\end{tabular}

La densidad relativa de cada muestra de PB se obtuvo mediante la aplicación de la norma ASTM D $854-02$ (2002), debido a que el procedimiento IRAM 1520 (2002) para agregados finos no garantiza una adecuada eliminación del aire ocluido en las partículas. En la Tabla 2 se presentan los valores de densidad relativa de los materiales caracterizados.

Tabla 2 - Densidad relativa de los materiales

\begin{tabular}{|c|c|c|c|c|c|c|}
\hline Material & Aditivo & Cemento & \multicolumn{4}{|c|}{ Polvo de basalto } \\
\hline Código & SP & CPC40 & CEN & CIE & CEL & CSM \\
\hline Densidad relativa & 1,09 & 2,96 & 2,97 & 2,91 & 2,91 & 3,00 \\
\hline
\end{tabular}

En cuanto al tamaño de partículas para el PB se determinó la relación en peso de la fracción pasante tamiz \#200 $(75 \mu \mathrm{m})$ respecto de la fracción pasante tamiz \#100 (150 $\mu \mathrm{m})$ aplicando el método especificado en la norma IRAM 1641 (1983), con modificaciones en el procedimiento: la muestra a analizar se obtuvo directamente de la fracción pasante tamiz \#8 de la arena de trituración; la presión de lavado se incrementó gradualmente hasta llegar a $0,6 \mathrm{daN} / \mathrm{cm}^{2}$ para evitar pérdida de material; el tiempo de duración del ensayo dependía de la presencia de material pasante tamiz \#200 en la bandeja de lavado, duplicando en general el tiempo especificado por la norma. Los porcentajes obtenidos para las muestras analizadas se exponen en la Tabla 3 junto a valores de superficie específica, incluyendo valores para las dos submuestras (CEL-Sed y CSMCic).

Tabla 3 - Finura Blaine y porcentaje de material pasante tamiz \#200

\begin{tabular}{|c|c|c|c|c|c|c|}
\hline Parámetro & Unidad & CPC40 & CEN & CSM & CEL-Sed & CSM-Cic \\
\hline Superficie específica & $\mathrm{m}^{2} / \mathrm{kg}$ & 565 & 229 & 221 & 351 & 366 \\
\hline Pasante tamiz \#200 & $\%(\mathrm{p} / \mathrm{p})$ & 100 & 77 & 77 & 89 & 100 \\
\hline
\end{tabular}

\subsection{Estudio sobre pastas}

En función de los contenidos de PB presentes en la arena de trituración (18 a 34\% según Pizzutti, D. et al. (2016)) y los parámetros generales de diseño para HAC (Fava C. y Fornasier G. (2004)), se establecieron las series de pastas que se detallan en la Tabla 4, donde los códigos numéricos se corresponden respectivamente con la relación en volumen entre PB y el contenido total de MF (PB + cemento), y la relación en volumen entre agua y MF. La cantidad de SP se especifica en gramos por volumen de pasta elaborada (1000 ml).

El estudio del comportamiento de pastas autocompactantes en estado fresco consistió en evaluar parámetros directamente relacionados con la fluidez y viscosidad de las mezclas a través del ensayo de escurrimiento con mini cono de Kantro (Kantro, D.L. (1980)) y el ensayo de velocidad de flujo en cono de Marsh con orificio de $10 \mathrm{~mm}$. En el primero se obtiene un valor de Escurrimiento (en $\mathrm{mm}$ ) a partir de la diferencia entre el diámetro de extendido final -como promedio de 4 diámetros medidos a 45 - y la base del mini cono (38 mm), mientras que en el segundo se miden los tiempos en que fluyen 300 y $500 \mathrm{ml}$ de pasta de un total de $1000 \mathrm{ml}$ vertidos en el cono (T300 y T500).

Para evaluar el efecto sobre la fluidez y la demanda de aditivo, se elaboraron pastas con idénticas proporciones de materiales, pero con PB de diferente finura. Esto se evidencia en el porcentaje de material pasante tamiz \#200 de la Tabla 3. 
Tabla 4 - Pastas elaboradas

\begin{tabular}{|c|c|c|c|c|c|c|c|}
\hline Código & $\begin{array}{c}\text { Relación } \\
\text { PB/MF } \\
(\% v / v)\end{array}$ & $\begin{array}{c}\text { Relación } \\
\text { a/MF } \\
\text { (v/v) }\end{array}$ & $\begin{array}{c}\text { Cantidad de } \\
\text { SP } \\
\text { (gr/lt) }\end{array}$ & $\begin{array}{c}\text { Relación } \\
a / c \\
(p / p)\end{array}$ & $\begin{array}{c}\text { Dosis de } \\
\text { aditivo SP/c } \\
\text { (\%p/p) }\end{array}$ & $\begin{array}{c}\text { Muestra } \\
\text { de PB }\end{array}$ & $\begin{array}{c}\text { Pasante } \\
\text { tamiz \#200 } \\
(\% p / p)\end{array}$ \\
\hline P-20-80 & 20 & 0,8 & 8,3 a 13,7 & 0,32 & 0,6 a 1,0 & CEL-Sed & 89 \\
\hline P-20-80 & 20 & 0,8 & 13,3 & 0,32 & 1,0 & CSM-Cic & 100 \\
\hline P-20-100 & 20 & 1 & 4,8 a 7,4 & 0,40 & 0,4 a 0,6 & CEN & 77 \\
\hline P-20-100 & 20 & 1 & 9,9 & 0,40 & 0,75 & CEL-Sed & 89 \\
\hline P-33-62 & 33 & 0,62 & 20,2 & 0,30 & 1,65 & CEN & 77 \\
\hline P-33-80 & 33 & 0,8 & 10,1 & 0,40 & 0,9 & CSM-Cic & 100 \\
\hline P-33-83 & 33 & 0,83 & 4,5 a 8,5 & 0,40 & 0,4 a 0,75 & CEN & 77 \\
\hline P-33-100 & 33 & 1 & 4,1 a 6,7 & 0,48 & 0,4 a 0,65 & CEN & 77 \\
\hline P-33-100 & 33 & 1 & 5,0 & 0,50 & 0,5 & CSM-Cic & 100 \\
\hline P-43-62 & 43 & 0,62 & 17,3 a 19,4 & 0,35 & 1,6 a 1,8 & CEN & 77 \\
\hline P-43-72 & 43 & 0,72 & 6,2 a 8,2 & 0,40 & 0,6 a 0,8 & CEN & 77 \\
\hline P-50-54 & 50 & 0,54 & 16,9 а 19,9 & 0,35 & 1,7 a 2,0 & CEN & 77 \\
\hline P-50-62 & 50 & 0,62 & 10,5 a 24,4 & 0,40 & 1,1 a 2,6 & CEN & 77 \\
\hline P-50-62 & 50 & 0,62 & 66,7 & 0,40 & 7,4 & CEL-Sed & 89 \\
\hline P-50-80 & 50 & 0,8 & 5,2 а 6,9 & 0,51 & 0,6 a 0,8 & CEN & 77 \\
\hline P-50-80 & 50 & 0,8 & 8,6 a 13,7 & 0,51 & 1,0 a 1,65 & CEL-Sed & 89 \\
\hline P-50-80 & 50 & 0,8 & 11,7 a 15,2 & 0,53 & 1,4 a 1,8 & CSM-Cic & 100 \\
\hline P-50-100 & 50 & 1 & 1,9 a 5,4 & 0,64 & 0,25 a 0,7 & CEN & 77 \\
\hline P-50-100 & 50 & 1 & 3,1 a 4,7 & 0,64 & 0,4 a 0,6 & CEL-Sed & 89 \\
\hline P-50-100 & 50 & 1 & 5,3 a 5,5 & 0,64 & 0,7 a 0,75 & CSM-Cic & 100 \\
\hline
\end{tabular}

A fin de evaluar la pérdida de fluidez en el tiempo, se midieron en la serie de pastas P-50-62 los parámetros de escurrimiento y velocidad de flujo en 5 y 30 o 40 minutos desde el momento en que entran en contacto agua y cemento.

A modo de complemento, se evaluaron la resistencia a compresión y el módulo de rotura a flexión en pastas con distintos porcentajes de reemplazo (20/33/50\%) y a similares rangos de fluidez, aplicando procedimientos de la norma IRAM 1622 (2006). Se moldearon 3 (tres) probetas prismáticas de $40 \mathrm{~mm}$ por 40 $\mathrm{mm}$ por $160 \mathrm{~mm}$ para cada serie analizada.

Para evaluar el efecto sobre la fluidez y la demanda de aditivo, se elaboraron pastas con idénticas proporciones de materiales, pero con PB de diferente finura. Esto se evidencia en el porcentaje de material pasante tamiz \#200 de la Tabla 3.

A fin de evaluar la pérdida de fluidez en el tiempo, se midieron en la serie de pastas P-50-62 los parámetros de escurrimiento y velocidad de flujo en 5 y 30 o 40 minutos desde el momento en que entran en contacto agua y cemento.

A modo de complemento, se evaluaron la resistencia a compresión y el módulo de rotura a flexión en pastas con distintos porcentajes de reemplazo (20/33/50\%) y a similares rangos de fluidez, aplicando procedimientos de la norma IRAM 1622 (2006). Se moldearon 3 (tres) probetas prismáticas de $40 \mathrm{~mm}$ por 40 $\mathrm{mm}$ por $160 \mathrm{~mm}$ para cada serie analizada. 


\section{Resultados y discusión}

En la Tabla 5 se ordenan los resultados más relevantes de ensayos realizados sobre las pastas especificadas en la Tabla 4. Estos resultados son analizados con mayor detalle en las figuras presentadas.

Tabla 5 - Resultados de escurrimiento y velocidad de flujo para 5 minutos

\begin{tabular}{|c|c|c|c|}
\hline Pasta & $\begin{array}{c}\text { Escurr. } \\
\text { (mm) }\end{array}$ & $\begin{array}{c}\text { Vf300 } \\
\text { (ml/s) }\end{array}$ & $\begin{array}{c}\text { PT\#200 } \\
\text { (\%) }\end{array}$ \\
\hline P-20-80-10.5 & 132 & 9,0 & 89 \\
\hline P-20-100-7.4 & 131 & 31,5 & 77 \\
\hline P-33-62-20.2 & 130 & 9,2 & 77 \\
\hline P-33-83-7.5 & 131 & 12,8 & 77 \\
\hline P-33-100-6.7 & 130 & 31,2 & 77 \\
\hline P-50-54-19.5 & 129 & 3,8 & 77 \\
\hline P-50-62-10.5 & 119 & 3,3 & 77 \\
\hline P-50-62-11.5 & 131 & 4,0 & 77 \\
\hline P-50-62-12.4 & 137 & 4,9 & 77 \\
\hline P-50-62-14.3 & 151 & 4,6 & 77 \\
\hline P-50-62-24.4 & 151 & 4,9 & 77 \\
\hline P-50-80-5.2 & 108 & 7,6 & 77 \\
\hline P-50-80-6.0 & 133 & 9,9 & 77 \\
\hline P-50-80-6.9 & 142 & 11,4 & 77 \\
\hline
\end{tabular}

\begin{tabular}{|c|c|c|c|}
\hline Pasta & $\begin{array}{c}\text { Escurr. } \\
\text { (mm) }\end{array}$ & $\begin{array}{c}\text { Vf300 } \\
\text { (ml/s) }\end{array}$ & $\begin{array}{c}\text { PT\#200 } \\
\text { (\%) }\end{array}$ \\
\hline P-50-80-8.6 & 80 & 5,4 & 89 \\
\hline P-50-80-13.7 & 157 & 11,8 & 89 \\
\hline P-50-80-11.7 & 81 & 7,8 & 100 \\
\hline P-50-80-15.2 & 125 & 7,9 & 100 \\
\hline P-50-100-1.9 & 71 & 13,8 & 77 \\
\hline P-50-100-3.1 & 80 & 26,8 & 77 \\
\hline P-50-100-3.9 & 108 & 32,1 & 77 \\
\hline P-50-100-4.7 & 117 & 31,4 & 77 \\
\hline P-50-100-5.1 & 128 & 32,1 & 77 \\
\hline P-50-100-5.4 & 135 & 31,0 & 77 \\
\hline P-50-100-3.1 & 74 & 17,1 & 89 \\
\hline P-50-100-4.7 & 113 & 22,9 & 89 \\
\hline P-50-100-5.5 & 107 & 14,3 & 100 \\
\hline & & & \\
\hline
\end{tabular}

Uno de los parámetros más influyentes en la demanda de aditivo, para mantener un nivel de fluidez determinado, es la relación volumétrica entre agua y material fino (a/MF), como se puede apreciar en ambos gráficos de la Figura 1 para tres niveles de reemplazo de cemento por PB. La cantidad necesaria de aditivo SP para lograr aproximadamente $130 \mathrm{~mm}$ de escurrimiento, es creciente a relaciones a/MF por debajo de 0,80 , pero con menor incidencia en porcentajes de reemplazo altos (a). Mientras que la velocidad de flujo aumenta considerablemente a medida que se incrementa el agua disponible para movilizar el sistema de partículas en suspensión (b), aun cuando disminuya la cantidad de SP y siendo casi independiente por debajo de 0,80 de a/MF.
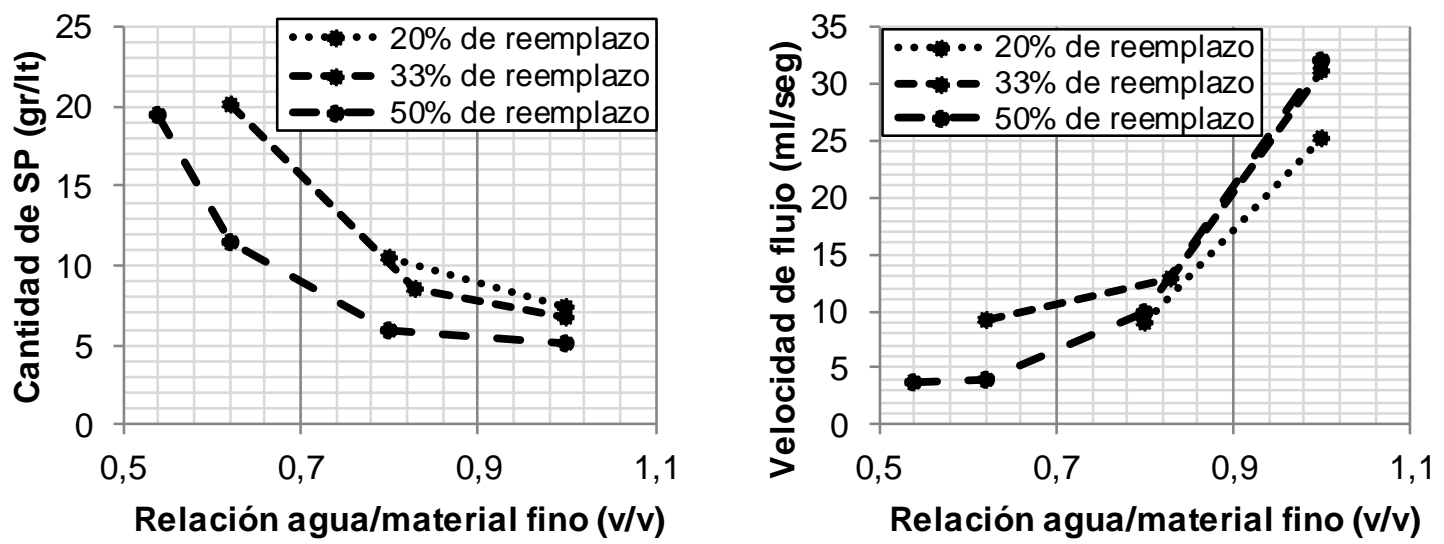

Figura 1 - (a) Demanda de aditivo y (b) velocidad de flujo para $130 \mathrm{~mm}$ de escurrimiento

En la Figura 2 se puede observar claramente el fenómeno de saturación del aditivo en pastas de las series P-50-62, P-50-80 y P-50-100 con idéntica finura de PB, donde aun cuando se incremente la cantidad de SP, tanto el escurrimiento (a) como la velocidad de flujo (b) no sufren variaciones importantes a partir de un valor determinado, el cual depende fuertemente de la relación volumétrica a/MF. Además, con valores de a/MF crecientes se puede obtener elevada velocidad de flujo (b), pudiendo lograrse mezclas menos viscosas. 

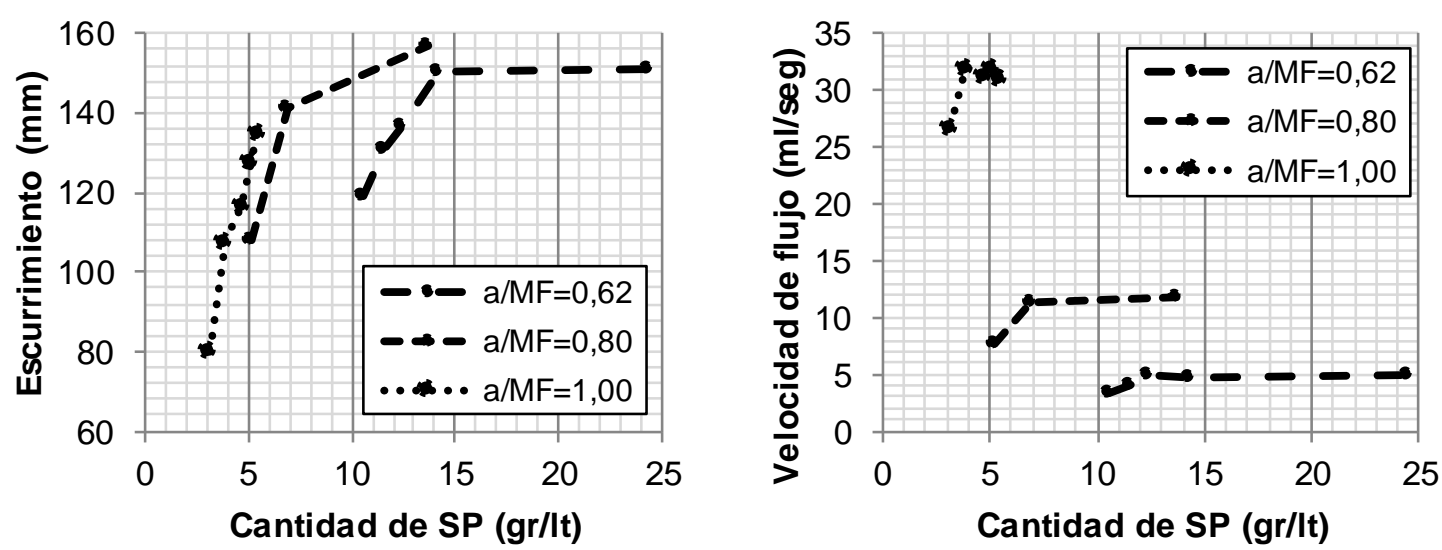

Figura 2 - (a) Escurrimiento y (b) velocidad de flujo según cantidad de aditivo

En el caso de la finura del PB, analizada en pastas de las series P-50-80 (Figura 3.a) y P-50-100 (Figura 3.b) y parametrizada mediante el porcentaje de MF pasante tamiz \#200, se aprecia una fuerte relación entre la demanda de aditivo y el porcentaje de finos menores a $75 \mu \mathrm{m}$ para mantener un mismo escurrimiento (a), mientras que la velocidad de flujo está más influenciada por la finura y la cantidad de SP que el escurrimiento (b). La muestra de PB con mayor finura, correspondiente al polvo de cantera CSM-Cic (PT\#200=100\%), requiere una excesiva cantidad de aditivo para alcanzar una fluidez adecuada cuando el porcentaje de reemplazo es elevado (50\%).
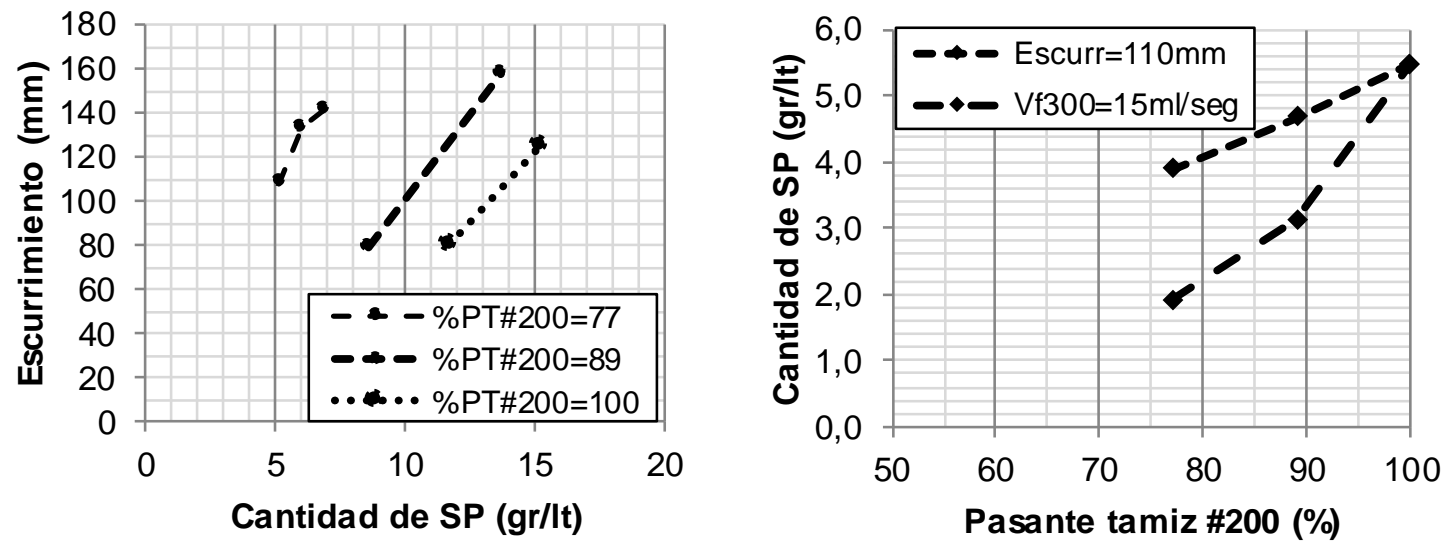

Figura 3 - (a) Escurrimiento y (b) cantidad de aditivo según porcentaje de PT\#200

En la Tabla 6 se comparan los resultados de escurrimientos y velocidad de flujo para distintos tiempos medidos sobre pastas de la serie P-50-62 con cantidades crecientes de SP. Al respecto, se observa que la pérdida de fluidez al transcurrir el tiempo es mayor para bajas cantidades de aditivo, al igual que la disminución en la velocidad de flujo.

Tabla 6 - Resultados de escurrimiento y velocidad de flujo para 5, 30 y 40 minutos

\begin{tabular}{|c|c|c|c|c|c|c|}
\hline \multirow{2}{*}{ Pasta } & \multicolumn{4}{|c|}{ Escurrimiento (mm) } & \multicolumn{3}{c|}{ Velocidad de flujo Vf300 (ml/s) } \\
\cline { 2 - 7 } & $\mathbf{5}^{\prime}$ & $\mathbf{3 0}$ & $\mathbf{4 0}$ & $\mathbf{5}^{\prime}$ & $\mathbf{3 0}$ & $\mathbf{4 0}^{\prime}$ \\
\hline P-50-62-10.5 & 119 & 99 & - & 3,3 & 0,0 & - \\
\hline P-50-62-12.4 & 137 & 117 & - & 4,9 & 3,0 & - \\
\hline P-50-62-14.3 & 151 & 151 & - & 4,6 & 4,6 & - \\
\hline P-50-62-24.4 & 151 & - & 150 & 4,9 & - & 4,2 \\
\hline
\end{tabular}

En referencia a la resistencia mecánica, según datos de la Tabla 7 y Figura 4, se observa una disminución de resistencia a porcentajes elevados de reemplazo (a), tanto en la resistencia a compresión como en el módulo de rotura a flexión evaluados según IRAM 1622 (2006). Se aprecia una correlación prácticamente lineal entre la relación a/c y la resistencia a compresión (b), alejándose de la típica curva de Abrams para hormigones convencionales. El efecto de dilución podría afectar la resistencia en porcentajes de reemplazo elevados. 
Tabla 7 - Resistencias mecánicas

\begin{tabular}{|c|c|c|c|}
\hline Parámetros & P-20-100-4.8 & P-33-100-5 & P-50-100-5.3 \\
\hline Porcentaje de reemplazo (\%) & 20 & 33 & 50 \\
\hline Relación a/c $(p / p)$ & 0,40 & 0,50 & 0,64 \\
\hline Resistencia a compresión (MPa) & 53,3 & 43,4 & 26,8 \\
\hline Módulo de rotura a flexión (MPa) & 8,4 & 7,0 & 6,3 \\
\hline
\end{tabular}
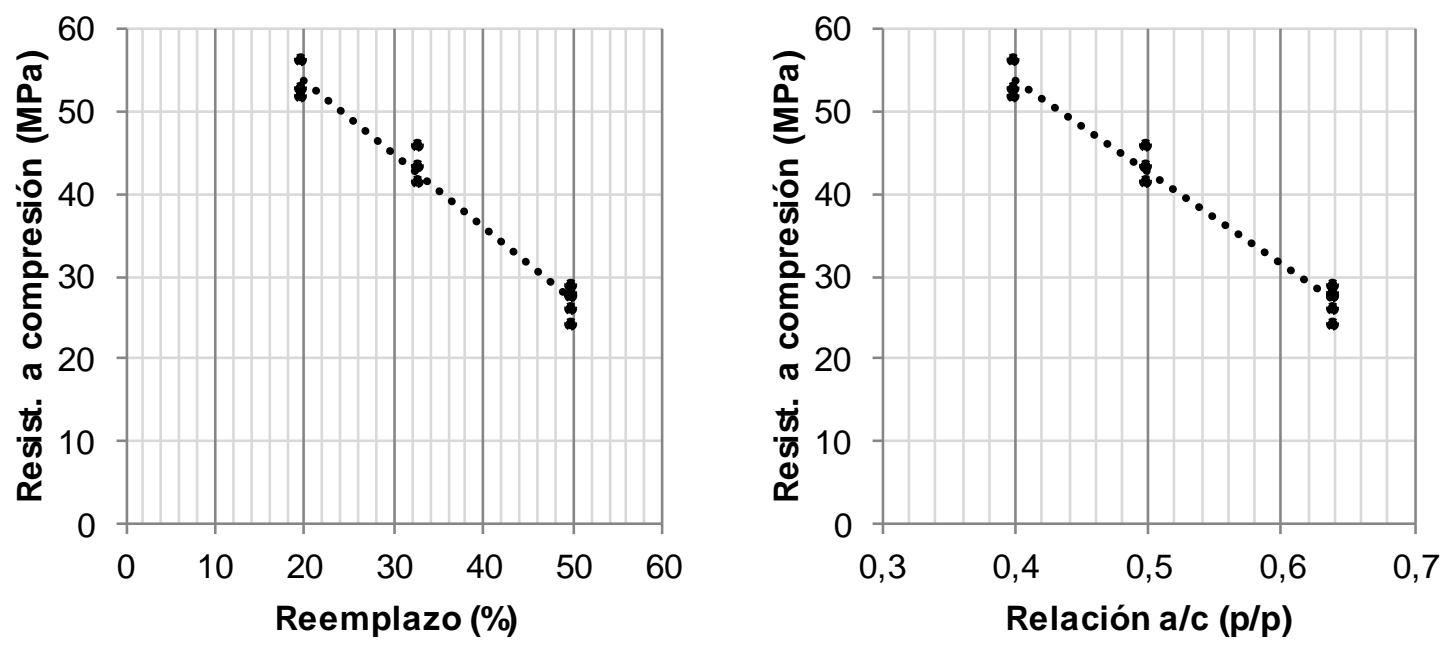

Figura 4 - (a) Resistencia a compresión según el porcentaje de reemplazo y (b) Correlación entre la resistencia a compresión y la relación a/c.

\section{Conclusiones}

$\checkmark$ La densidad relativa del PB es muy similar a la del cemento y se recomienda que se obtenga mediante la aplicación de la norma ASTM D 854-02 (2002) para garantizar una adecuada eliminación del aire ocluido.

$\checkmark$ La relación volumétrica entre agua y material fino (a/MF) es el principal factor que influye en la demanda de SP, en el punto de saturación del aditivo y en la fluidez y viscosidad de las pastas de cemento con diferentes porcentajes de reemplazo con PB.

$\checkmark$ Por debajo de valores de relación a/MF cercanos a 0,8, la autocompactabilidad se torna antieconómica, incrementando excesivamente la cantidad de aditivo necesaria para garantizar fluidez y viscosidad adecuadas. En cambio, para valores del entorno de 1,0, es evidente la mejora en la velocidad de flujo de las pastas con PB. Sin embargo, el riesgo de segregación podría limitar el incremento de a/MF para los distintos porcentajes de reemplazo.

$\checkmark$ La finura del PB afecta fuertemente la demanda de SP para lograr características de fluidez adecuadas, siendo más influyente si se desea mantener una velocidad de flujo determinada. Por ello, resulta importante una adecuada caracterización de la finura del PB para vincularla con parámetros de diseño como la relación a/MF y la cantidad necesaria de SP.

$\checkmark$ Para reducir la pérdida de fluidez a lo largo del tiempo es importante determinar una cantidad mínima de SP que garantice el sostenimiento del efecto reductor de agua sin favorecer la segregación de la mezcla.

$\checkmark$ Desde al punto de vista de las resistencias mecánicas, aun con porcentajes altos de reemplazo, podrían lograrse morteros $u$ hormigones de resistencia moderada.

$\checkmark$ Si bien el PB contiene un volumen importante de material fino que podría favorecer el efecto "filler" en pastas de cemento, la granulometría del PB podría generar pérdida de resistencia a compresión o tracción por efecto de dilución, problemática que se evidencia en el decreciendo progresivo que se observa para relaciones a/c por encima de 0,50. 


\section{Agradecimientos}

Este trabajo ha sido desarrollado gracias al apoyo y financiamiento de la Facultad de Ingeniería de la Universidad Nacional de Misiones (UNaM), poniendo a disposición la infraestructura, el equipamiento y el personal de apoyo técnico que forma parte del Laboratorio de Ingeniería Civil (LIC). Además, en referencia a ensayos de granulometría de partículas y superficie específica, se agradece el aporte del Laboratorio de Micro-partículas (Facultad de Ingeniería de la UNCPBA), el Laboratorio de Entrenamiento Multidisciplinario para la Investigación Tecnológica (LEMIT) y el LEMaC Centro de Investigaciones Viales (UTN Regional La Plata).

\section{Referencias Bibliográficas}

ASTM D 854 -02, (2002), Standard test methods for specific gravity of soil solids by water pycnometer.

Benyamina S.; Menadi B.; Kamali-Bernard S.; Kenai S., (2019), Performance of self-compacting concrete with manufactured crushed sand, Advances in Concrete Construction, 7, 87-96.

Cepuritis R.; Jacobsen S.; Pedersen B.; Mørtsell E., (2011), Crushed sand in concrete - Effect of particle shape in different fractions and filler properties on rheology, Construction and Building Materials, 33, 771-776.

Cepuritis R., Jacobsen S., Smeplass S., Mørtsell E.; Wigum B. J., (2016), Micro-proportioning of SCC with crushed aggregate: Rheology of filler modified cement pastes, Proceedings 8th International RILEM Symposium on Self-Compacting Concrete, 8, 3-13.

Dobiszewska M., (2017), Waste materials used in making mortar and concrete, Journal of Materials Education, 39, 133-156.

Dobiszewska M. y Beycioğlu A., (2017), Investigating the Influence of Waste Basalt Powder on Selected Properties of Cement Paste and Mortar, IOP Conference series: Materials Science and Engineering, (245).

Dobiszewska M.; Pichór W.; Szołdra P., (2019), Effect of basalt powder addition on properties of mortar, MATEC Web of Conferences (262).

Fava C. y Fornasier G., (2004), Hormigón autocompactante, De Hormigones especiales, Asociación Argentina de Tecnología del Hormigón, La Plata, (2004), 57-96.

Felekoğlu, B., (2008), A comparative study on the performance of sands rich and poor in fines in selfcompacting concrete, Construction and Building Materials, 22, 646-654.

IRAM 1520, (2002), Agregados finos: Métodos de laboratorio para la determinación de la densidad relativa real, de la densidad relativa aparente y de la absorción de agua.

IRAM 1622, (2006), Cemento Portland: Determinación de resistencias mecánicas.

IRAM 1624, (2005), Cemento: Método de ensayo para la determinación de la densidad.

IRAM 1641, (1983), Material de relleno ("filler") para mezclas asfálticas: Método de ensayo de finura, por tamizado húmedo.

IRAM 1663, (2002), Hormigón de cemento: Aditivos químicos.

Kantro, D.L., (1980), Influence of water-reducing admixtures on properties of cement paste: A miniature slump test, Cement, Concrete and Aggregates, 2, 2, 95-102.

Mahalingam B.; Sreehari P.; Rajagopalan S.; Ramana Gopal S.; Mohammed Haneefa K., (2018), Mechanical characterization and robustness of self-compacting concrete with quarry dust waste and class-f fly ash as fillers, Advances in Materials and Metallurgy. Lecture Notes in Mechanical Engineering, 365-373.

Pizzutti, D.; Rivero, C.; Reich, G.; Klimczuk, M.; Detke, F., (2016), Distribución granulométrica en pilas de arena de trituración basáltica, VII Congreso Internacional y 21a Reunión Técnica de la Asociación Argentina de Tecnología del Hormigón, Salta, Argentina, septiembre 2016.

Revilla-Cuesta V.; Skaf M.; Faleschini F.; Manso J. M.; Ortega-López V., (2020), Self-compacting concrete manufactured with recycled concrete aggregate: An overview, Journal of Cleaner Production, 262.

Schankosk R. A., Pilar R., Prudêncio L. R. Jr.; Ferron R. D., (2016), Evaluation of quarry powders as viscositymodifying material in cement mixtures, 8th International RILEM Symposium on Self-Compacting Concrete, 8, (2016), 187-196. 
Shi C.; Wu Z.; Lv K.; Wu L., (2015), A review on mixture design methods for self-compacting concrete, Construction and building materials, 84, 387-398.

Tobes J. M., (2009), Hormigones autocompactantes simples y reforzados con fibras: diseño, caracterización y aplicaciones, Tesis doctoral, Universidad Nacional de La Plata.

Uysal M. y Yilmaz K., (2011), Effect of mineral admixtures on properties of self-compacting concrete, Construction and Building Materials, 33, 771-776. 